\title{
Excreted/Secreted Proteins from Trypanosome Procyclic Strains
}

\author{
Celestine Michelle Atyame Nten, ${ }^{1,2}$ Nicolas Sommerer, ${ }^{3}$ Valerie Rofidal, ${ }^{3}$ Christophe Hirtz,, 4 \\ Michel Rossignol, ${ }^{3}$ Gerard Cuny, ${ }^{1}$ Jean-Benoit Peltier, ${ }^{3}$ and Anne Geiger ${ }^{1}$
}

${ }^{1}$ UMR 177, IRD-CIRAD, CIRAD TA A-17 / G, Campus International de Baillarguet, 34398 Montpellier Cedex 5, France

${ }^{2}$ UMR, CNRS 5554, Institut des Sciences de l'Evolution, Université Montpellier II, 34095 Montpellier Cedex 5, France

${ }^{3}$ Organisation des Nations Unies, INRA, UR1199, LPF; 2 place Pierre Viala Bât., 1334060 Montpellier Cedex 01, France

${ }^{4}$ UFR Odontologie, EA 4203, 545 Avenue du Pr Viala, Université Montpellier I, 34193 Montpellier Cedex 5, France

Correspondence should be addressed to Anne Geiger, anne.geiger@mpl.ird.fr

Received 22 July 2009; Accepted 19 September 2009

Academic Editor: Luis I. Terrazas

Copyright ( $\odot 2010$ Celestine Michelle Atyame Nten et al. This is an open access article distributed under the Creative Commons Attribution License, which permits unrestricted use, distribution, and reproduction in any medium, provided the original work is properly cited.

\begin{abstract}
Trypanosoma secretome was shown to be involved in parasite virulence and is suspected of interfering in parasite life-cycle steps such as establishment in the Glossina midgut, metacyclogenesis. Therefore, we attempted to identify the proteins secreted by procyclic strains of T. brucei gambiense and T. brucei brucei, responsible for human and animal trypanosomiasis, respectively. Using mass spectrometry, 427 and 483 nonredundant proteins were characterized in T. brucei brucei and T. brucei gambiense secretomes, respectively; $35 \%$ and $42 \%$ of the corresponding secretome proteins were specifically secreted by T. brucei brucei and T. brucei gambiense, respectively, while 279 proteins were common to both subspecies. The proteins were assigned to 12 functional classes. Special attention was paid to the most abundant proteases (14 families) because of their potential implication in the infection process and nutrient supply. The presence of proteins usually secreted via an exosome pathway suggests that this type of process is involved in trypanosome ESP secretion. The overall results provide leads for further research to develop novel tools for blocking trypanosome transmission.
\end{abstract}

\section{Introduction}

Tsetse flies are vectors of African trypanosomes, the causative agents of sleeping sickness in humans and nagana in animals. Following a long period of increasing prevalence, human African trypanosomiasis now seems to be decreasing [1]. However, this debilitating disease still affects a wide range of people in sub-Saharan Africa [2] and is invariably fatal if untreated. Nagana is estimated to cost African agriculture US $\$ 4.5$ billion per year [3]. Until now, drugs have been unsatisfactory, some being toxic and all difficult to administer [1]. Furthermore, resistance to drugs is increasing [4]. Therefore, the search for novel strategies must continue and among them are alternative vector-based strategies [5]. These strategies require a clear and full understanding of the various steps and mechanisms involved in the transmission of the parasite. To be transmitted, trypanosomes must undergo cycles of development of varying complexity within the tsetse fly, transforming from bloodstream forms to procyclic nonmammalian infective forms in the fly midgut (establishment). To complete their life cycle, Trypanosoma brucei brucei and the causative agents of human African trypanosomiasis, Trypanosoma brucei rhodesiense, and Trypanosoma brucei gambiense must migrate from the midgut to the salivary glands where they transform into infective metacyclic forms. The fly's ability to acquire the parasite, favor its maturation, and transmit it to a mammalian host is known as vector competence, which depends on complex interactions between Glossina, the parasite, and the environment. The factors involved in establishment are largely unknown and those involved in maturation are unclear. Nevertheless, among factors involved in vector competence appear to be the sex of the fly $[6,7]$, the trypanosome genotype $[8]$, the tsetse intestinal lectin $[9,10]$, and the tsetse immune responses [11]. More recently, antioxidants have been shown to greatly increase midgut trypanosome infection rates in tsetse [12], suggesting that oxidative stress plays a role in the refractoriness of tsetse to trypanosome 
infection. Moreover, a NO signal has been suggested to be required to promote the trypanosome migration to the salivary glands and its subsequent maturation into mammalian infective forms [13]. Different Glossina species exhibit large differences in vector competence $[14,15]$. Nevertheless, vectorial competence of a given Glossina species could also be influenced by the nature of the parasite species [16]. Moreover, differences in the establishment and maturation rates have even been observed for different genotypes belonging to a given parasite subspecies [17]. These observations demonstrate the complexity of the mechanisms governing parasite development in the tsetse fly. Given that knowledge on the infection process that remains limited, further studies are required to characterize the overall Glossina-parasite molecular interactions.

The identification of the proteins secreted by the insect procyclic parasite may contribute to the increasing of the understanding of the infection process and lead to the identification of potential targets for drug and/or vaccine design.

In this respect, we have investigated the excreted/secreted proteins (ESPs) produced by two procyclic Trypanosoma brucei parasite subspecies (T. brucei brucei and T. brucei gambiense) and compared their respective secretomes.

\section{Materials and Methods}

2.1. Procyclic Parasite Cultures. STIB 215 [18] and Biyamina $[19,20]$ procyclic forms of Trypanosoma brucei brucei and Trypanosoma brucei gambiense, respectively, were grown at $25^{\circ} \mathrm{C}$, in Cunningham's medium supplemented with $20 \%$ fetal calf serum. When the cultures displayed logarithmic growth, procyclic parasites were washed four times with phosphate buffer saline (PBS) and spun for 10 minutes for $2600 \mathrm{~g}$ at $25^{\circ} \mathrm{C}$.

2.2. Excreted/Secreted Protein (ESP) Production. The parasites were resuspended at a concentration of $2 \cdot 10^{8}$ cells $/ \mathrm{mL}$ in a secretion buffer [21] and incubated for 2 hours at $25^{\circ} \mathrm{C}$ for ESP production. During the incubation process, the parasite cell viability was controlled every 15 minutes by flow cytofluorometry using the DNA intercalant propidium iodide (IP) procedure. Briefly, $2 \cdot 10^{6}$ parasites were incubated with $1 \mu \mathrm{g}$ IP in $1 \mathrm{~mL}$ PBS as recommended by the manufacturer (Immunotech, Marseille, France). The cells were immediately analyzed with a FACScan flow cytometer (Becton Dickinson, Ivry, France) using an argon-ion laser. Cellular integrity was assessed by microscopic examination. After the 2-hour incubation, the secretion of ESPs was stopped by centrifugation of the parasites, $2600 \mathrm{~g}$ for 10 minutes at $4{ }^{\circ} \mathrm{C}$. The supernatant was collected and filtered on $0.2-\mu \mathrm{m}$ filter and immediately mixed with protease inhibitors. The ESPs were then concentrated by ultrafiltration on a PM - 10 (10 KDa cut-off) membrane (Amicon) for further protein electrophoretic separation (SDS-PAGE). The protein concentration was determined by the Bradford dye binding procedure (Bio-Rad), and $300 \mu \mathrm{g}$ of ESPs were further separated using SDS-PAGE.
2.3. Total Proteome Preparation. Parasite pellets were resuspended at a concentration of $2 \cdot 10^{8}$ cells $/ \mathrm{mL}$ of laemmli buffer. The protein concentration was determined, and $100 \mu \mathrm{g}$ of proteins of the total proteomes were further separated using SDS-PAGE.

2.4. One-Dimensional Electrophoretic Analysis. Proteins from the different samples (ESPs and total proteome) were heated at $100^{\circ} \mathrm{C}$ for 2 minutes and spun for 5 minutes for $14.000 \mathrm{~g}$ prior to separation using one-dimensional SDS-PAGE. Proteins were separated on $24 \times 18 \mathrm{~cm}$ Tricine/SDS/ureapolyacrylamide gels (12\% acrylamide) [22]. After migration, the gels were fixed, and the proteins were visualized using coomassie brilliant blue R-250. Pictures of the gels were taken with an Amersham Biosciences Personal Densitometer (BioRad).

2.5. Protein Identification by Mass Spectrometry. Sixty four protein spots were excised manually from the onedimensional gels of ESPs, washed, digested with trypsin, and extracted with formic acid. Protein digests were analyzed using either a triple-quadrupole mass spectrometer (QTRAP 4000; Applied Biosystems), coupled to a nanochromatography system (Dionex) or an ion trap mass spectrometer (Esquire HCT; Bruker), and interfaced with an HPLCChip system (Agilent). MS/MS data were searched against NCBI and Trypanosoma brucei databases using Mascot software. Raw data were analyzed using Data Analysis software (Bruker) to generate a peak list for searching a Trypanosoma database extracted from the Sanger Institute. The Mascot (v2.2) search engine was used with the following parameters: one missed cleavage allowed for trypsin, carboxymethylation of cyst as fixed modification, methionine oxidation as variable modification, and a 0.6-Da tolerance range for mass accuracy in MS/MS. At least one matching sequence of tags of high quality was needed for positive identification of proteins. Potential false-positive identifications have been addressed as described by Elias et al., (2005) [23], using identical search parameters against a database in which the sequences have been reversed. We set a false discovery rate (FDR) of 1\%. When the Mascot peptide score was below (and even above) the Mascot peptide score indicated for an FDR of $1 \%$, a systematic manual validation was done with stringent parameters (at least $6 \mathrm{y}$ or b ions, at least 4 consecutive ions, and peptidic sequence formed of more than 7 amino acids). The proteins were classified according to MapMan (http://mapman.gabipd.org/).

\section{Results}

The main objective of the study was a comprehensive characterization of the proteins secreted by two different strains of T. brucei in procyclic form, T. brucei gambiense/Biyamina strain (whose bloodstream form is pathogenic for humans) and T. brucei brucei/Stib 215 strains (whose bloodstream form is pathogenic for animals). A three-step investigation was conducted and consisted in the following: (1) onedimensional electrophoresis profiling of the secreted proteins 


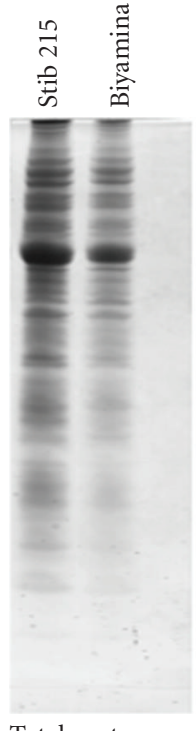

Total proteomes



Secretomes

Figure 1: Protein profile of two different T. brucei strains. Coomassie blue-stained SDS-PAGE showing (from right to left) the marker (PM), secretome, and proteome from two procyclic Trypanosoma strains: Biyamina and Stib 215.

and comparison with the corresponding proteomes, (2) the identification of the secreted proteins by mass spectrometry and distribution into functional classes, and (3) a crosscomparative analysis between (a) the secretomes of the two strains (procyclic forms) and (b) the secretome of the procyclic forms and the proteome.

3.1. One-Dimensional Electrophoresis Protein Profiling of the Secretomes and Proteomes. The profiles generated by the electrophoretic separation of the secretome proteins from the two procyclic parasite strains (Biyamina and Stib 215) and their corresponding proteomes are shown in Figure 1. A high number of bands were separated, corresponding to proteins with molecular weights ranging from 7.2 to $205.8 \mathrm{kDa}$.

Clear differences were noted between the electrophoretic profiles of the proteome of the two procyclic strains and their corresponding secretome profiles, in terms of both proteic band intensity and presence or absence of several protein bands. Furthermore, since over the entire 2-hour secretion process (a) the parasites' viability remained constant and was greater than $98 \%$ and (b) the incubated trypanosomes presented normal morphology and motility, all these data indicate that the secretome resulted from an active export of proteins from living trypanosomes and not from cellular lysis.

The electrophoretic profiles of the proteome of the two procyclic strains show that they have many protein bands in common (32-34 visible bands); some differences in intensity were noted after coomassie staining.

Visual observation of the 1D gels showed similarities as well as some quantitative and qualitative differences between the secretome profiles of the two parasite subspecies.



Figure 2: Classification of T. brucei proteins from two different procyclic strains into functional categories. Proteins from the two strains (Biyamina and Stib 215) were classified into 12 functional categories. The $x$-axis shows the following categories: (1) unassigned function, (2) folding and degradation, (3) nucleotide metabolism, (4) carbohydrate metabolism, (5) amino acid metabolism, (6) protein synthesis, (7) signaling, (8) cell cycle and organization, (9) lipid and cofactor, (10) transport, (11) redox, and (12) RNA/DNA metabolism. The $y$-axis shows the percentage of each category for each strain.

3.2. Identification of the Secreted Proteins and Grouping into Functional Categories. Secreted proteins from procyclic strains were fractionated by 1D SDS-PAGE, and 64 spots were selected all along the $1 \mathrm{D}$ gel for MS/MS analysis. This resulted in the identification of 427 secreted proteins from the Stib 215 (T. brucei brucei) strain and 483 proteins from Biyamina (T. brucei gambiense). Supplementary table 1 (in supplementary material available online at doi:10.1155/2010/212817) lists the proteins identified. They were classified into 12 main functional categories (Figure 2 and Supplementary table 1). Both of the procyclic strains' secretomes, the proteins associated with unassigned functions, those associated with (un)folding and degradation processes, and protein synthesis were quantitatively the largest categories, with a total of $60 \%$ of the secreted proteins (Figure 2). Moreover, lipid and cofactor, transport, RNA/DNA metabolism, cell cycle and organization, nucleotide metabolism, and amino acid metabolism contribute approximately $26 \%$ of the secreted proteins, and finally carbohydrate metabolism accounts for about $9 \%$ of the secreted proteins. Globally, the importance (expressed as the percentage of the proteins concerned) of each functional class is similar for both procyclic strains, except for the redox and signaling classes that are under-represented in the $T$. brucei brucei strain (Stib 215) secretome as compared with the T. brucei gambiense strain (Biyamina) (Figure 2).

3.3. Overlap between the Secretomes of the Two Procyclic Parasites. Comparing the secretomes of two procyclic strains (Figure 3) showed 279 common secreted proteins $(57.8 \%$ and $65.3 \%$ of the Biyamina and the Stib 215 ESPs, resp.), $204(42.2 \%)$ were specifically secreted by T. brucei gambiense (Biyamina), and $148(34.7 \%)$ were secreted by T. brucei 


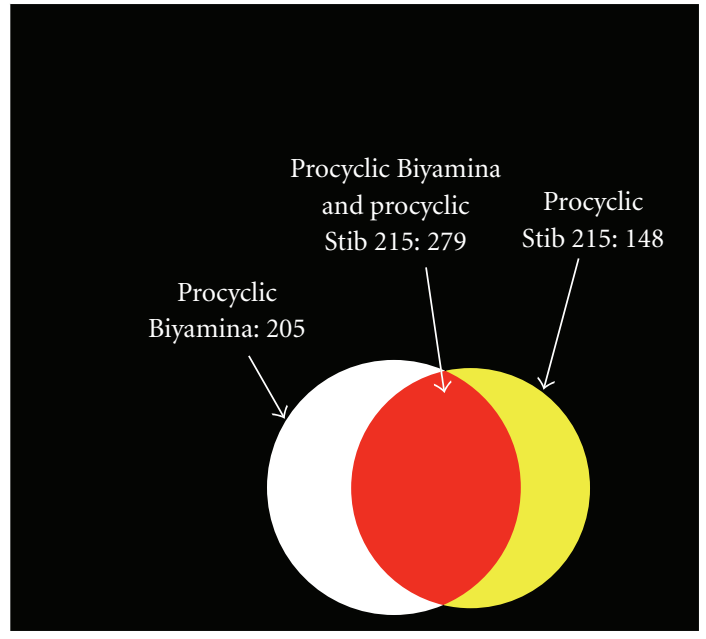

Figure 3: Overlap between secretomes of Biyamina and Stib procyclic strains. Proteins found in the analysis of Biyamina and Stib secretomes were compared. The red domain represents the proteins common to the two strains ( 279 proteins).

TABle 1: Peptidase families found in the secretome of procyclic forms of parasites.

\begin{tabular}{lccc}
\hline & Stib 215 & Biyamina & Common \\
\hline Metallopeptidase, family M16 & + & & \\
Cysteine peptidase, family C65 & + & & \\
Serine peptidase, family S10 & & + & \\
Major surface protease gp63, & & + & \\
family M8 & + & \\
Cysteine peptidase, family C1 & + & \\
Metallopeptidase, family M32 & & & + \\
Cysteine peptidase, family C2 & & + \\
Serine peptidase, family S9A & & & + \\
Aminotripeptidase, family S8 & & + \\
Metallopeptidase, family M3 & & + \\
Metallopeptidase, family M24 & & & + \\
Metallopeptidase, family M17 & & & + \\
Metallopeptidase, family M1 & & & + \\
Peptidase (M20/M25/M40 & & \\
Family) & & + \\
\hline
\end{tabular}

brucei (Stib 215). The proteins specifically secreted by either Stib 215 or Biyamina were represented in all 12 functional categories previously identified. However, the (un)folding and degradation protein class was particularly valuable because it grouped 14 different families of peptidases; four of which were specifically secreted by the T. brucei gambiense procyclic strain (Biyamina) and two others by T. brucei brucei (Stib 215). The other eight secreted peptidase families were common to both strains (Table 1). Surprisingly, we also identified a serine protease inhibitor among the proteins secreted by both strains.

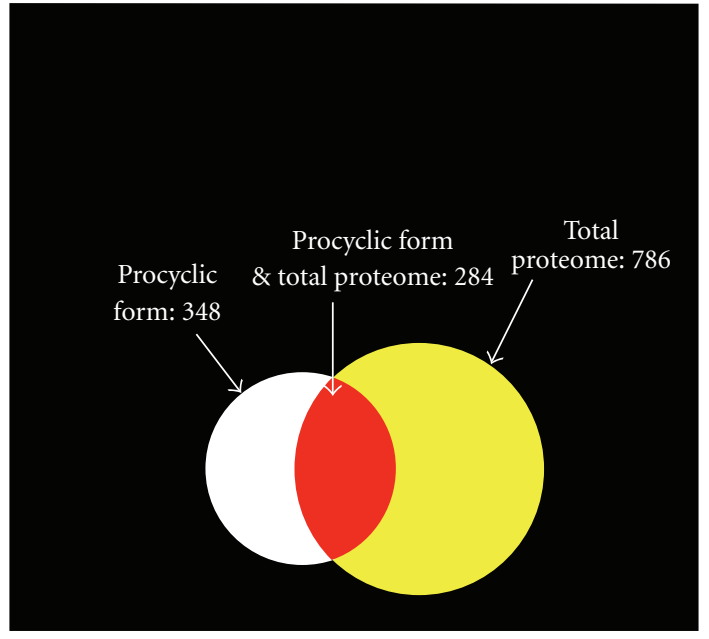

Figure 4: Overlap between all the proteins from procyclic secretomes (Biyamina and Stib) and total proteome [59]. Proteins found in the analysis of secretomes and T. brucei total proteome were compared. The red domain represents the proteins common in the two proteomes (284 proteins).

More than one-third of the proteins (39.2\%) specifically secreted by Stib 215 procyclic strain corresponds to hypothetical proteins, although they account for only $27.8 \%$ in Biyamina. Of major interest was the observation that some proteins, such as calreticulin and IgE-dependent histamine-releasing factor which belong to the class of signaling proteins, were specifically secreted only by the Biyamina strain; this strain also secreted higher levels (2.9\%) of proteins belonging to the redox class.

3.4. Overlap between the Secretome of Procyclic Parasites and Total Proteome. Finally, only $45 \%$ of the proteins secreted by the procyclic strains were common to the proteins from the procyclic total proteome (Figure 4). This data also corroborates that the secretome results from an active protein exportation from living cells.

\section{Discussion}

The life cycle of Trypanosoma is complex and requires the expression of specialized proteins for the development of the parasite in both invertebrate and vertebrate host environments, to escape host immune responses.

For successful Glossina infection, the parasite has to adapt to its novel environment; it must switch both from a homoiothermic to a poikilothermic host and from a mammal to an insect. Furthermore, it must develop new weapons or choose among its panel of weapons to fight a novel type of host defense.

In vertebrate hosts, trypanosome bloodstream forms are protected by the variant surface glycoprotein (VSG), which prevents their recognition by the host immune system. When the parasites are taken up by the insect in the blood meal, they must confront the insect's immune system, mainly 
composed of midgut proteases, physical barriers such as peritrophic matrix [24], reactive oxygen species [25, 26], and antimicrobial peptides [27]. A number of studies have revealed that the procyclins, surface glycoproteins expressed by trypanosome procyclic forms, may protect these forms against tsetse midgut proteases $[28,29]$. However, there are yet no examples of direct interactions between tsetse fly and trypanosome molecules. The purpose of this study was to identify the whole excreted/secreted proteins in two trypanosome procyclic forms to better understand the interactions between the trypanosome and tsetse fly in terms of establishment in the midgut or vectorial competence and to search for new tools for vector control strategies.

We successfully used 1D electrophoresis and MS/MS to establish the first extensive protein map of the secretome of the procyclic form of the parasite using stringent mass spectrometry criteria to validate potential candidates (False Discovery Rate $(\mathrm{FDR})<1 \%)$. The substantial differences between the set of proteins secreted by both trypanosome strains and those of their respective total proteomes demonstrate that the proteins identified in the secretome are indeed secreted and are not an artifact resulting from possible cell lysis during the secretion step. Sequencing 1D gel showed that a large number of proteins were specifically secreted by both Biyamina (T. brucei gambiense) and Stib 215 (T. brucei brucei) $(42.4 \%$ and $34.6 \%$, resp.). The differences in the protein secretion profiles between the two parasite subspecies may reflect their genetic differences and could possibly be related to differences in Glossina vector competence [14, 15, 30-34].

To better understand the possible role of the secreted proteins, they were assigned to functional groups. The groups of unfolding and degradation proteins, protein synthesis, and proteins with no assigned functions were the most numerous.

Unknown or hypothetical proteins are a source of candidates that could be used to explore novel functions.

We did not expect to find so many different peptidase families among the unfolding and degradation classes of secreted proteins. Members of serine, cysteine proteinases, and metallopeptidases were identified. The group of proteases deserves the greatest attention because these enzymes cover a large panel of physiological and pathological functions. Consequently, representatives of this group are known to be virulence factors, to favor parasite invasion and its growth in the hostile host environment, to make it possible to escape the host immune defenses, and/or, finally, to produce nutrients by hydrolyzing host proteins. Some parasites such as Plasmodium falciparum produce a diversified panel of secreted and surface-bound proteases [35]. In a variety of animals, including humans [36] as well as arthropods [37], the blood clotting system plays an important role in the immobilization of invading parasites, and in preventing their dispersal from the site of initial invasion. Proteolytic degradation on the blood clots would allow escape from this confinement. How the parasite proteases process to inactivate or cause the inappropriate expression of host immune defense systems is a major question. In addition to their direct role as virulence factors, secreted proteases are involved in various housekeeping functions during parasitic infection. As shown in Porphyromonas gingivalis, the proteolytic degradation of host proteins is able to produce nutritive elements for parasite growth [38]. Virulence has correlated positively with the expression of secreted proteases in a variety of systems where virulence can be modified genetically or in response to environmental cues. As a result, strains of Leishmania mexicana, engineered by targeted gene disruption of the genes encoding a pair of cysteine proteases, showed attenuated virulence [39]. The virulence of laboratory strains and clinical isolates of Entamoeba histolytica correlates well with the level of activity of a secreted cysteine protease [40]. In addition, inhibitors of serine proteases have been shown to block the entry of merozoites of Plasmodium into erythrocytes [41].

Surprisingly, an inhibitor of serine proteases (ecotin) has been discovered in the secretion products of trypanosome strains. The wide distribution of serine protease inhibitors, and their ability to regulate a variety of divergent proteinasedependent physiological functions, shows that they are closely involved in a host of biological processes [42]. In several systems, serine protease inhibitors from viruses have been implicated in pathogen escape from the host immune system [43]. In Brugia malayi, cysteine-protease inhibitors that can inhibit host proteases involved in antigen processing have been discovered [44].

Besides contributing to protect the parasite against the host's defense mechanisms, many proteases show the capacity to induce perturbations in the host physiology. In addition to their protein degrading activity, proteases perform highly specific processing tasks that can affect protein structure, function, life span, and localization. By limited and specific cleavage, proteases can act as switches, turning protein activity on or off; they can also modulate protein function in more complex ways, regulating vital processes. Metallopeptidase of the M32 family has been found in the secretome of procyclic parasite. This family is absent in eukaryotic genomes other than trypanosomatids [45]. Consequently, proteases constitute both promising candidates for developing diagnostic tools and attractive drug targets to fight trypanosomes and subsequently to control sleeping sickness.

Proteins involved in signaling make up another group of proteins identified in the secretome of procyclic trypanosome, but even this is not a major class; some proteins, such as calreticulin, could play physiopathological roles. Autoantibodies against calreticulin are found in the sera of human hosts in a number of parasitic diseases [46] and it was suggested that the parasite-derived calreticulin could trigger an inappropriate immune response against selfantigens through molecular mimicry [47].

The class of proteins involved in protein synthesis is quantitatively $(17 \%)$ well represented in the secretome. The role of such secreted proteins in the tsetse fly midgut is currently unknown. However, some proteins, such as elongation factor- 1 , were suggested to be a virulence factor in Leishmania [48]. 
We did not expect to find so many representatives of the carbohydrate metabolism protein class $(9.79 \%$ in the T. brucei gambiense secretome and $8.27 \%$ in the $T$. brucei brucei secretome). Several papers have reported on the ability of the procyclic cell to adapt its metabolism to different environments. Because tsetse hemolymph contains abundant supplies of amino acids, and because the tsetse fly itself uses proline as a major carbon source during flight [49], it has long been speculated that in situ, the procyclic form of trypanosomes, might use proline. Accordingly, proline is a key component in the culture media currently used to cultivate the parasite. In contrast, another study has shown that, when grown in high glucose-concentrated media, the procyclic cells are susceptible to glycolytic inhibitors and to the downregulation of genes encoding glycolytic enzymes. This confirms the flexibility of the trypanosome metabolism and its adaptability to diverse growth conditions. Cells grown under chemostat conditions were shown to regulate their glucose metabolism according to the availability of proline [50]. Since the buffer we used to stimulate protein secretion contained sugar, it was not surprising to find a high level of enzymes involved in carbohydrate metabolism. Besides, procyclic forms establish mainly in the vector's midgut where sugar may be abundantly available immediately after a blood meal. Nevertheless, two major questions remain unresolved because glycolytic enzymes are expected to be intracellular enzymes. (1) Why is there such a high level of carbohydrate metabolism enzymes secreted? (2) What is the role of such enzymes when secreted in the fly's midgut?

The redox-protein class of secreted proteins was poorly represented. This finding was also unexpected because in the flies' midgut, reactive oxygen species are generated during immune responses or because of the abundance of heme molecules freed from the digested blood meal [51]. In addition, some of these proteins may play roles other than the detoxification of reactive oxygen. Therefore, a superoxide dismutase (SOD) has been shown to be secreted by T. cruzi; it was suggested that this enzyme was involved in both the parasite defense mechanism and the establishment of the parasite in the host $[52,53]$. In another context, SOD was considered to participate in the immune escape in filarial infections $[54,55]$.

Finally, in the procyclic parasite secretome, we have identified 13 (GAPDH, clathrin heavy chain, Rab protein, ubiquitin, 14-3-3 proteins, cyclophilin, enolase, hsp70, actin, cofilin, tubulin $\alpha$ and $\beta$, and histone) out of the 22 proteins commonly associated with the exosome secretion pathway in various organisms [56]. Moreover, we have also identified translationally controlled tumor protein (TCTP) in this secretome. This protein was shown to be present in exosomes $[57,58]$.

These results suggest that the secretome proteins may be secreted via an exosome pathway that has never been described until now in trypanosomes. This also suggests that exosome-directed transmission-blocking vaccines could be developed and are capable of suppressing the capacity of the parasite to infect the vector and consequently preventing the spread of the parasite in human populations.

\section{Conclusions}

To conclude, the Trypanosoma parasite is a multistage organism that evolves in tsetse flies and vertebrates. Designing drugs that persistently interrupt the life cycle of this parasite requires a comprehensive understanding of its biology and the mechanism involving the vector-parasite interactions. The primary goal of our proteomic investigation was to identify secretome proteins of procyclic parasites to improve the knowledge of these interactions. The secretome encompasses a spectrum of proteins that may be required for every facet of the parasite's lifestyle, from the modification of the physiological environment to the immune escape. This may open leads to initiate novel strategies for controlling the parasites and sleeping sickness.

\section{Acknowledgments}

We sincerely thank Eric Bellard for assistance with the bioinformatic software and Dr. Philippe Vincendeau and Dr. Philippe Holzmuller for providing secretion buffer.

\section{References}

[1] M. P. Barrett, "The rise and fall of sleeping sickness," The Lancet, vol. 367, no. 9520, pp. 1377-1378, 2006.

[2] WHO, "Human African trypanosomiasis (sleeping sickness): epidemiological update," The Weekly Epidemiological Record, vol. 81, no. 8, pp. 71-80, 2006.

[3] E. Reinhardt, "Travailler ensemble : la mouche tsé-tsé et la pauvreté rurale," Chronique ONU, ONU Editor, September 2002, http://www.un.org/french/pubs/chronique/2002/ numero2/0202p17_la_mouche_tsetse.html.

[4] E. Matovu, T. Seebeck, J. C. K. Enyaru, and R. Kaminsky, "Drug resistance in Trypanosoma brucei spp., the causative agents of sleeping sickness in man and nagana in cattle," Microbes and Infection, vol. 3, no. 9, pp. 763-770, 2001.

[5] R. V. M. Rio, Y. Hu, and S. Aksoy, "Strategies of the hometeam: symbioses exploited for vector-borne disease control," Trends in Microbiology, vol. 12, no. 7, pp. 325-336, 2004.

[6] C. Dale, S. C. Welburn, I. Maudlin, and P. J. M. Milligan, "The kinetics of maturation of trypanosome infections in tsetse," Parasitology, vol. 111, no. 2, pp. 187-191, 1995.

[7] P. J. M. Milligan, I. Maudlin, and S. C. Welburn, “Trypanozoon: infectivity to humans is linked to reduced transmissibility in tsetse. II. Genetic mechanisms," Experimental Parasitology, vol. 81, no. 3, pp. 409-415, 1995.

[8] S. C. Welburn, I. Maudlin, and P. J. M. Milligan, "Trypanozoon: infectivity to humans is linked to reduced transmissibility in tsetse. I. Comparison of human serum-resistant and human serum-sensitive field isolates," Experimental Parasitology, vol. 81, no. 3, pp. 404-408, 1995.

[9] I. Maudlin and S. C. Welburn, "Lectin mediated establishment of midgut infections of Trypanosoma congolense and Trypanosoma brucei in Glossina morsitans," Tropical Medicine and Parasitology, vol. 38, no. 3, pp. 167-170, 1987.

[10] I. Maudlin and S. C. Welburn, "The role of lectins and trypanosome genotype in the maturation of midgut infections in Glossina morsitans," Tropical Medicine and Parasitology, vol. 39, no. 1 , pp. 56-58, 1988. 
[11] C. $\mathrm{Hu}$ and S. Aksoy, "Innate immune responses regulate trypanosome parasite infection of the tsetse fly Glossina morsitans morsitans," Molecular Microbiology, vol. 60, no. 5, pp. 1194-1204, 2006.

[12] E. T. MacLeod, I. Maudlin, A. C. Darby, and S. C. Welburn, "Antioxidants promote establishment of trypanosome infections in tsetse," Parasitology, vol. 134, no. 6, pp. 827-831, 2007.

[13] E. T. Macleod, A. C. Darby, I. Maudlin, and S. C. Welburn, "Factors affecting trypanosome maturation in tsetse flies," PLoS ONE, vol. 2, no. 2, article e239, 2007.

[14] S. K. Moloo and S. B. Kutuza, "Comparative study on the infection rates of different laboratory strains of Glossina species by Trypanosoma congolense," Medical and Veterinary Entomology, vol. 2, no. 3, pp. 253-257, 1988.

[15] S. K. Moloo and S. B. Kutuza, "Comparative study on the susceptibility of different Glossina species to Trypanosoma brucei brucei infection," Tropical Medicine and Parasitology, vol. 39, no. 3, pp. 211-213, 1988.

[16] I. Maudlin, "Inheritance of susceptibility to Trypanosoma congolense infection in Glossina morsitans," Annals of Tropical Medicine and Parasitology, vol. 76, no. 2, pp. 225-227, 1982.

[17] S. Ravel, D. Patrel, M. Koffi, V. Jamonneau, and G. Cuny, "Cyclical transmission of Trypanosoma brucei gambiense in Glossina palpalis gambiensis displays great differences among field isolates," Acta Tropica, vol. 100, no. 1-2, pp. 151-155, 2006.

[18] W. C. Gibson, T. F. de C Marshall, and D. G. Godfrey, "Numerical analysis of enzyme polymorphismml: a new approach to the epidemiology and taxonomy of trypanosomes of the subgenus Trypanozoon," Advances in Parasitology, vol. 18 , pp. $175-246,1980$.

[19] F. Mathieu-Daude, A. Bicart-See, M.-F. Bosseno, S.-F. Breniere, and M. Tibayrenc, "Identification of Trypanosoma brucei gambiense group I by a specific kinetoplast DNA probe," The American Journal of Tropical Medicine and Hygiene, vol. 50, no. 1, pp. 13-19, 1994.

[20] D. G. Godfrey, C. M. Scott, W. C. Gibson, D. Mehlitz, and U. Zillmann, "Enzyme polymorphism and the identity of Trypanosoma brucei gambiense," Parasitology, vol. 94, no. 2, pp. 337-347, 1987.

[21] P. Holzmuller, D. G. Biron, P. Courtois, et al., "Virulence and pathogenicity patterns of Trypanosoma brucei gambiense field isolates in experimentally infected mouse: differences in host immune response modulation by secretome and proteomics," Microbes and Infection, vol. 10, no. 1, pp. 79-86, 2008.

[22] H. Schägger and G. Von Jagow, "Tricine-sodium dodecyl sulfate-polyacrylamide gel electrophoresis for the separation of proteins in the range from 1 to $100 \mathrm{kDa}$," Analytical Biochemistry, vol. 166, no. 2, pp. 368-379, 1987.

[23] J. E. Elias, W. Haas, B. K. Faherty, and S. P. Gygi, "Comparative evaluation of mass spectrometry platforms used in large-scale proteomics investigations," Nature Methods, vol. 2, no. 9, pp. 667-675, 2005.

[24] M. J. Lehane, "Peritrophic matrix structure and function," Annual Review of Entomology, vol. 42, pp. 525-550, 1997.

[25] Y. Hu and S. Aksoy, "An antimicrobial peptide with trypanocidal activity characterized from Glossina morsitans morsitans," Insect Biochemistry and Molecular Biology, vol. 35, no. 2, pp. 105-115, 2005.

[26] Z. Hao, I. Kasumba, and S. Aksoy, "Proventriculus (cardia) plays a crucial role in immunity in tsetse fly (Diptera: Glossinidiae)," Insect Biochemistry and Molecular Biology, vol. 33, no. 11, pp. 1155-1164, 2003.
[27] Z. Hao, I. Kasumba, M. J. Lehane, W. C. Gibson, J. Kwon, and S. Aksoy, "Tsetse immune responses and trypanosome transmission: implications for the development of tsetsebased strategies to reduce trypanosomiasis," Proceedings of the National Academy of Sciences of the United States of America, vol. 98, no. 22, pp. 12648-12653, 2001.

[28] A. Acosta-Serrano, E. Vassella, M. Liniger, et al., "The surface coat of procyclic Trypanosoma brucei: programmed expression and proteolytic cleavage of procyclin in the tsetse fly," Proceedings of the National Academy of Sciences of the United States of America, vol. 98, no. 4, pp. 1513-1518, 2001.

[29] M. Liniger, A. Acosta-Serrano, J. Van Den Abbeele, et al., "Cleavage of trypanosome surface glycoproteins by alkaline trypsin-like enzyme(s) in the midgut of Glossina morsitans," International Journal for Parasitology, vol. 33, no. 12, pp. 13191328, 2003.

[30] P. Dukes, A. Kaukas, K. M. Hudson, T. Asonganyi, and J. K. Gashumba, "A new method for isolating Trypanosoma brucei gambiense from sleeping sickness patients," Transactions of the Royal Society of Tropical Medicine and Hygiene, vol. 83, no. 5, pp. 636-639, 1989.

[31] J. M. Harley and A. J. Wilson, "Comparison between Glossina morsitans, G. pallidipes and G. fuscipes as vectors of trypanosomes of the Trypanosoma congolense group: the proportions infected experimentally and the numbers of infective organisms extruded during feeding," Annals of Tropical Medicine and Parasitology, vol. 62, no. 2, pp. 178-187, 1968.

[32] J. M. Kazadi, Interactions between vector and trypanosome in determining the vectorial competence of tsetse flies, Ph.D. dissertation, University of Liège, Liège, Belgium, 2000.

[33] J. M. Reifenberg, D. Cuisance, J. L. Frezil, G. Cuny, and G. Duvallet, "Comparison of the susceptibility of different Glossina species to simple and mixed infections with Trypanosoma (Nannomonas) congolense savannah and riverine forest types," Medical and Veterinary Entomology, vol. 11, no. 3, pp. 246-252, 1997.

[34] D. Richner, R. Brun, and L. Jenni, "Production of metacyclic forms by cyclical transmission of West African Trypanosoma (T.) brucei isolates from man and animals," Acta Tropica, vol. 45, no. 4, pp. 309-319, 1988.

[35] J. H. McKerrow, E. Sun, P. J. Rosenthal, and J. Bouvier, "The proteases and pathogenicity of parasitic protozoa," Annual Review of Microbiology, vol. 47, pp. 821-853, 1993.

[36] O. D. Rotstein, "Role of fibrin deposition in the pathogenesis of intraabdominal infection," European Journal of Clinical Microbiology \& Infectious Diseases, vol. 11, no. 11, pp. 10641068, 1992.

[37] V. Isakova and P. B. Armstrong, "Imprisonment in a deathrow cell: the fates of microbes entrapped in the Limulus blood clot," The Biological Bulletin, vol. 205, no. 2, pp. 203-204, 2003.

[38] H. K. Kuramitsu, "Proteases of Porphyromonas gingivalis: what don't they do?" Oral Microbiology and Immunology, vol. 13, no. 5, pp. 263-270, 1998.

[39] J. Alexander, G. H. Coombs, and J. C. Mottram, "Leishmania mexicana cysteine proteinase-deficient mutants have attenuated virulence for mice and potentiate a Th1 response," The Journal of Immunology, vol. 161, no. 12, pp. 6794-6801, 1998.

[40] S. Reed, J. Bouvier, A. S. Pollack, et al., "Cloning of a virulence factor of Entamoeba histolytica. Pathogenic strains possess a unique cysteine proteinase gene," The Journal of Clinical Investigation, vol. 91, no. 4, pp. 1532-1540, 1993.

[41] C. B. Breton, T. Blisnick, H. Jouin, et al., "Plasmodium chabaudi p68 serine protease activity required for merozoite 
entry into mouse erythrocytes," Proceedings of the National Academy of Sciences of the United States of America, vol. 89, no. 20, pp. 9647-9651, 1992.

[42] X. Zang, A. K. Atmadja, P. Gray, et al., "The serpin secreted by Brugia malayi microfilariae, Bm-SPN-2, elicits strong, but short-lived, immune responses in mice and humans," The Journal of Immunology, vol. 165, no. 9, pp. 5161-5169, 2000.

[43] S. Kettle, A. Alcamí, A. Khanna, R. Ehret, C. Jassoy, and G. L. Smith, "Vaccinia virus serpin B13R(SPI-2) inhibits interleukin- $\beta$-converting enzyme and protects virus-infected cells from TNF- and Fas-mediated apoptosis, but does not prevent IL-1 $\beta$-induced fever," Journal of General Virology, vol. 78, no. 3, pp. 677-685, 1997.

[44] B. Manoury, W. F. Gregory, R. M. Maizels, and C. Watts, "Bm-CPI-2, a cystatin homolog secreted by the filarial parasite Brugia malayi, inhibits class II MHC-restricted antigen processing," Current Biology, vol. 11, no. 6, pp. 447-451, 2001.

[45] G. Niemirowicz, F. Parussini, F. Agüero, and J. J. Cazzulo, “Two metallocarboxypeptidases from the protozoan Trypanosoma cruzi belong to the M32 family, found so far only in prokaryotes," Biochemical Journal, vol. 401, no. 2, pp. 399-410, 2007.

[46] L. A. Rokeach, P. A. Zimmerman, and T. R. Unnasch, "Epitopes of the Onchocerca volvulus RAL1 antigen, a member of the calreticulin family of proteins, recognized by sera from patients with onchocerciasis," Infection and Immunity, vol. 62, no. 9, pp. 3696-3704, 1994.

[47] M. Dupuis, E. Schaerer, K.-H. Krause, and J. Tschopp, "The calcium-binding protein calreticulin is a major constituent of lytic granules in cytolytic T lymphocytes," Journal of Experimental Medicine, vol. 177, no. 1, pp. 1-7, 1993.

[48] D. Nandan, T. Yi, M. Lopez, C. Lai, and N. E. Reiner, "Leishmania EF- $1 \alpha$ activates the Src homology 2 domain containing tyrosine phosphatase SHP-1 leading to macrophage deactivation," Journal of Biological Chemistry, vol. 277, no. 51, pp. 50190-50197, 2002.

[49] E. Bursell, "Aspects of the metabolism of amino acids in the tsetse fly, Glossina (Diptera)," Journal of Insect Physiology, vol. 9, no. 4, pp. 439-452, 1963.

[50] B. H. Ter Kuile, "Adaptation of metabolic enzyme activities of Trypanosoma brucei promastigotes to growth rate and carbon regimen," Journal of Bacteriology, vol. 179, no. 15, pp. 46994705, 1997.

[51] M. F. Oliveira, B. L. Timm, E. A. Machado, et al., "On the prooxidant effects of haemozoin," FEBS Letters, vol. 512, no. 1-3, pp. 139-144, 2002.

[52] M. E. Villagrán, C. Marín, I. Rodríguez-Gonzalez, J. A. De Diego, and M. Sánchez-Moreno, "Use of an iron superoxide dismutase excreted by Trypanosoma cruzi in the diagnosis of chagas disease: seroprevalence in rural zones of the state of Queretaro, Mexico," The American Journal of Tropical Medicine and Hygiene, vol. 73, no. 3, pp. 510-516, 2005.

[53] M. Kabiri and D. Steverding, "Identification of a developmentally regulated iron superoxide dismutase of Trypanosoma brucei," Biochemical Journal, vol. 360, no. 1, pp. 173-177, 2001.

[54] S. Batra, R. K. Chatterjee, and V. M. L. Srivastava, "Antioxidant enzymes in Acanthocheilonema viteae and effect of antifilarial agents," Biochemical Pharmacology, vol. 40, no. 10, pp. 2363 2369, 1990.

[55] L. Tang, X. Ou, K. Henkle-Dührsen, and M. E. Selkirk, "Extracellular and cytoplasmic CuZn superoxide dismutases from Brugia lymphatic filarial nematode parasites," Infection and Immunity, vol. 62, no. 3, pp. 961-967, 1994.
[56] C. Olver and M. Vidal, "Proteomic analysis of secreted exosomes," Sub-Cellular Biochemistry, vol. 43, pp. 99-131, 2007.

[57] N. Amzallag, B. J. Passer, D. Allanic, et al., "TSAP6 facilitates the secretion of translationally controlled tumor protein/histamine-releasing factor via a nonclassical pathway," Journal of Biological Chemistry, vol. 279, no. 44, pp. 4610446112, 2004.

[58] A. Lespagnol, D. Duflaut, C. Beekman, et al., "Exosome secretion, including the DNA damage-induced p53-dependent secretory pathway, is severely compromised in TSAP6/Steap3null mice," Cell Death \& Differentiation, vol. 15, no. 11, pp. 1723-1733, 2008.

[59] A. Jones, A. Faldas, A. Foucher, et al., "Visualisation and analysis of proteomic data from the procyclic form of Trypanosoma brucei," Proteomics, vol. 6, no. 1, pp. 259-267, 2006. 

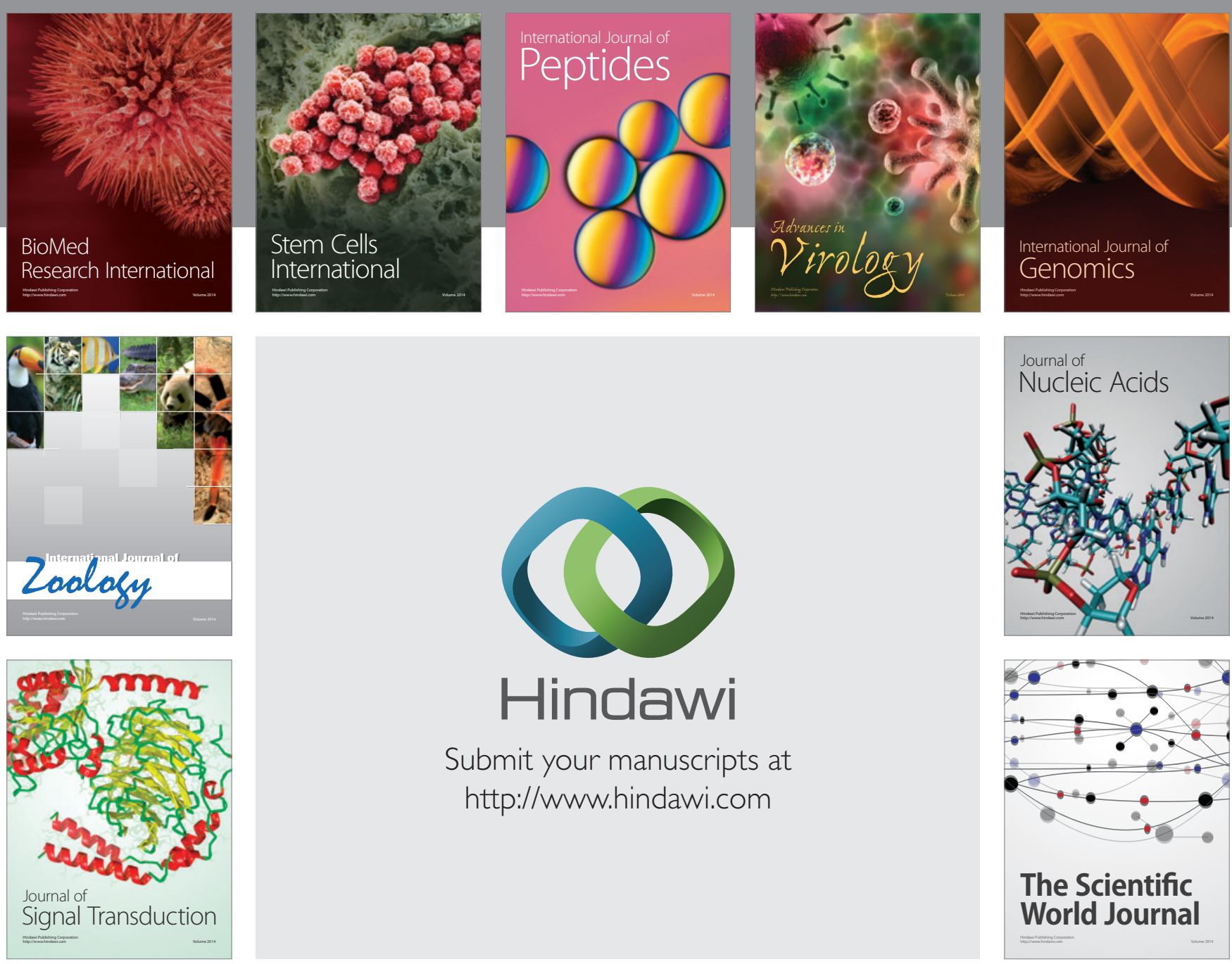

Submit your manuscripts at

http://www.hindawi.com


The Scientific World Journal
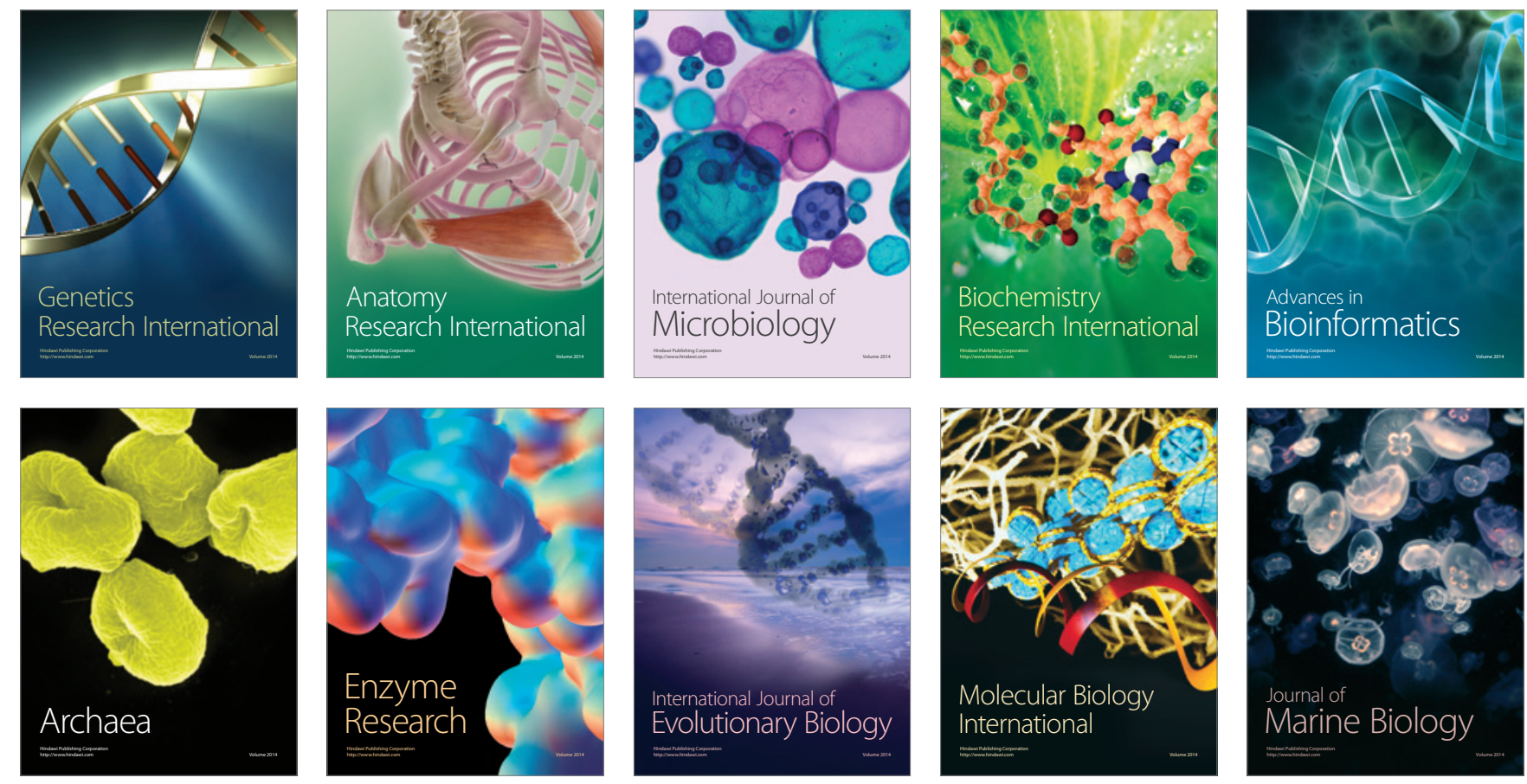